\title{
Genome-Wide association study of quantitative biomarkers identifies a novel locus for alzheimer's disease at 12p12.1
}

\author{
Brian Lee ${ }^{1}$, Xiaohui Yao ${ }^{1}$, Li Shen ${ }^{1 *}$ and for the Alzheimer's Disease Neuroimaging Initiative ${ }^{2}$
}

\begin{abstract}
Background: Genetic study of quantitative biomarkers in Alzheimer's Disease (AD) is a promising method to identify novel genetic factors and relevant endophenotypes, which provides valuable information to deconvolute mechanistic complexity and better understand disease subtypes.

Results: Using the data from the Alzheimer's Disease Neuroimaging Initiative (ADNI), we performed a genome-wide association study (GWAS) between 565,373 single nucleotide polymorphisms (SNPs) and 16 key AD biomarkers from 1,576 subjects at four visits. We identified a novel locus rs5011804 at 12p12.1 significantly associated with several AD biomarkers, including three cognitive traits (CDRSB, FAQ, ADAS13) and one imaging trait (fusiform volume). Additional mediation and interaction analyses investigated the relationships among this SNP, relevant biomarkers, and clinical diagnosis, confirming and further elaborating the genetic effects seen in the GWAS.

Conclusion: Our GWAS not only affirms key AD genes but also suggests the promising role of the SNP rs5011804 due to its associations with several AD cognitive and imaging outcomes. The SNP rs5011804 has a reported association with adult asthma and slightly affects intracranial volume but has not been associated with AD before. Our novel findings contribute to a more comprehensive view of the molecular mechanism behind AD.
\end{abstract}

Keywords: Alzheimer's disease, Genome-wide association study, Quantitative biomarkers, Cognitive traits, Imaging traits

\section{Background}

Alzheimer's disease (AD) is a complex neurodegenerative disease commonly characterized by memory impairments, cognitive decline, and the presence of both tau and $\mathrm{A} \beta[1]$. There is an urgent need for developing effective strategies to discover new AD risk or protective biomarkers for disease modeling and drug development [2]. Genetics plays an important role in AD with estimated heritability in the range of 58-79\% [3-5]. Genome wide association studies (GWAS) of case-control status have only discovered about 30 independent genetic factors

*Correspondence: li.shen@pennmedicine.upenn.edu

${ }^{1}$ Department of Biostatistics, Epidemiology and Informatics, Perelman School

of Medicine at the University of Pennsylvania, Philadelphia, USA

Full list of author information is available at the end of the article for AD susceptibility [6-8], which could not explain all the heritability and thus requires scientists to explore alternative search strategies for $\mathrm{AD}$ genetic determinants. With the availability of large-scale genetics, imaging, cognition and biomarker data in landmark studies such as the Alzheimer's Disease Neuroimaging Initiative (ADNI) [9-11], genetic analysis of multidimensional quantitative traits $(\mathrm{QT})$ in $\mathrm{AD}$ becomes an emerging and rapidly growing research field [12-14]. The QT approach has distinct advantages in power over categorical diagnoses. For example, genetic studies of AD imaging QTs have yielded some prominent new findings [15-19], including a few contributions to genetically based drug targets [18-21].

Specifically, there are a variety of cognitive, imaging and other biomarkers that can serve as AD-associated 
QTs, such as Clinical Dementia Rating - Sum of Boxes (CDRSB), Functional Activities Questionnaire (FAQ), and the Alzheimer's Disease Assessment Scale 13-item Cognitive Subscale (ADAS13) and neuroimaging volumetric measurements (e.g., those of fusiform and entorhinal cortex measured by the FreeSurfer (FS) software) [22]. As many of these measurements have been shown to accurately depict some form of mild or severe cognitive impairment related with dementia [23-26], it is possible to find a specific single nucleotide polymorphism (SNP) highly associated with $\mathrm{AD}$ by finding an association between the SNP and one or more indications of cognitive impairments/dementia as determined by these measurements.

Finding associations between SNPs and AD as a whole using specific QTs as a proxy is extremely useful for a couple of reasons. First, these measurable biological properties are often (much) more strongly associated to the pathogenesis of $\mathrm{AD}$ as a whole than a single, static diagnosis might be. Additionally, these quantitative continuous variables can account for and depict an individual's $A D$ status and neurological health more finely than a single diagnosis can, and possibly implicate a disease subtype mechanism. Given the heterogeneity of $\mathrm{AD}$, with only one diagnosis code representing the wide range of mild/intermediate cognitive impairments in addition to the study's case-control design, this single measure cannot offer the same amount of insight into an individual's progression status that a QT in the form of a measurable biomarker can, making the many measurements and calculations performed significantly less precise and powerful. Lastly, these QTs are continuous measures and statistically more powerful than case-control status, often requiring much fewer samples for a genetic discovery. This realization is fueled by the assumption that imaging, cognitive and other QTs are closer to the inherent neurobiology of the disease than a diagnosis itself; a previous study [27] has confirmed this assumption in showing there are instances where common genetic variation shows a stronger impact on brain structure than on risk for neuropsychiatric disorders. As such, biologically relevant variants that might not pass stringent multiple-test corrections in the typical case-control studies described here are more likely to be found in an associative study using intermediate biomarkers like this one [28].

Previous studies using data from the ADNI cohort highlighted several key AD genes including APOE, TOMM40, APOC1, BIN1, and CR1 [13] using AD diagnostic data. However, it is possible such studies might have missed some biologically relevant variants. Other studies have replicated these findings using $\mathrm{AD}$ neuroimaging data (including but not limited to [29-31]), fluid biomarkers (including [32-34]), or cognitive biomarkers (including $[35,36])$. However, not many studies have explicitly measured this larger spectrum of phenotypes across individuals within the same cohort. To bridge this gap, in this work, we perform GWAS analysis on a set of imaging, cognitive and biomarker QTs in ADNI, which are provided by the Quantitative Template for the Progression of AD (QT-PAD) Project (http://www.pi4cs.org/qtpad-challenge). The QT-PAD includes a set of longitudinal key AD biomarkers for $n=1,737$ ADNI participants. This large amount of normalized biomarker and detailed diagnosis data, when combined with covariates including age, gender and education level, allows researchers to perform significantly more powerful statistical analyses and directly compare GWAS results studying different QTs in $\mathrm{AD}$. Additionally, given the major role the time plays in $\mathrm{AD}$, the ability to study the progression of the disease over time and the corresponding genetic determinants is especially useful.

In summary, although previous GWAS have found various genetic variations that are highly associated with $A D$, it is possible that certain biologically significant variants that may not have survived the typical case-control study's corrected $p$ value thresholds. As such, in this work, we study a set of key AD QTs including a wide array of cognitive, cerebrospinal fluid (CSF), and imaging biomarkers involved in the QT-PAD project. Using this dataset allows for 1) increased statistical power compared with casecontrol GWAS studies; 2) the study of a wide variety of leading AD biomarkers, to help de-convolute mechanistic complexity and better understand disease subtypes; and 3) additional longitudinal analyses, to study the progression of the disease and the stability of the genetic determinants over time. Our overarching goal is to not only confirm the known $\mathrm{AD}$ genes but also identify novel $\mathrm{AD}$ genetic findings.

\section{Results}

\section{Targeted genetic association}

GWAS highlighted the effect of rs5011804 at 12p12.1 with several biomarker QTs across all four studied time points. The results of our analyses have been summarized below in two series of heat maps. These figures display all SNP-QT pairings across all four time points (ranging from the baseline visit to two years later) with low $p$-values, where significant pairings $\left(P<5 \times 10^{-8}\right.$, a genome-wide threshold) are marked with a red ' $X$ '. The first series (Fig. 1a through d) have age, gender, and education as covariates while the second series (Fig. 1e through h) consider age, gender, education, and genetic dosage of APOE $\epsilon 4$ as covariates. The analyses that do not control for APOE have 25 statistically significant SNP-outcome pairings across all four time points; the analyses that do control for APOE have 16 statistically significant SNPoutcome pairings across all four time points. $P$-values for the non-APOE analyses are as low as $1.879 \times 10^{-14}$ (association with CDRSB at the month 12 time point), 


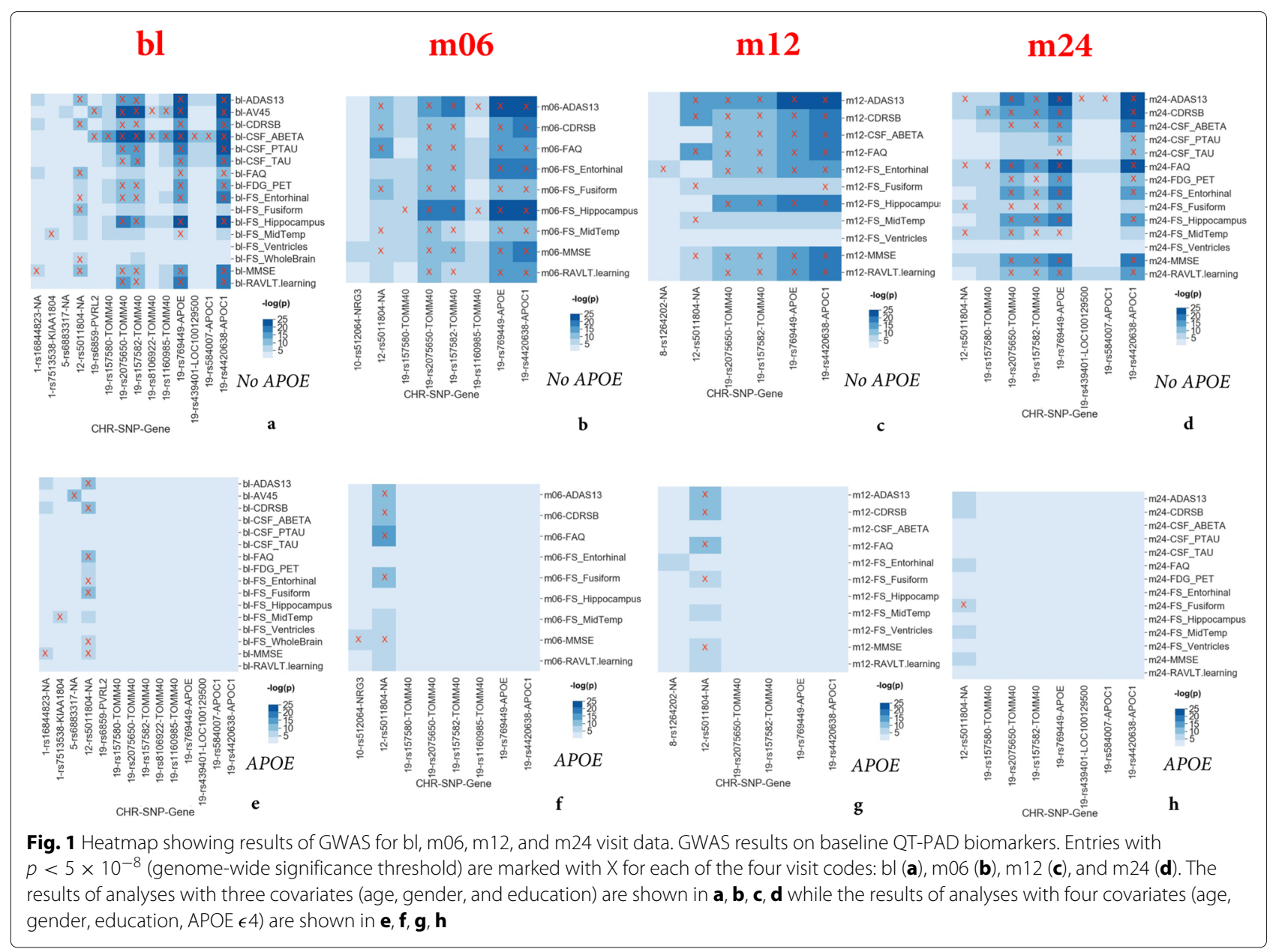

$2.163 \times 10^{-14}$ (association with FAQ at the month 12 time point), and $8.211 \times 10^{-14}$ (association with ADAS13 at the month 12 time point). $P$-values for the analyses that include APOE as a covariates are as low as $1.134 \times 10^{-13}$ (association with CDRSB at the month 12 time point), $1.262 \times 10^{-13}$ (association with FAQ at the month 12 time point), and $4.424 \times 10^{-13}$ (association with ADAS13 at the month 12 time point). One reassuring aspect of our analyses is that in addition to showing the significance of the novel SNP rs5011804 (Fig. 1), we have verified the significance of several variants on chromosome 19 strongly associated with $\mathrm{AD}$, including those from $\mathrm{AD}$ genes APOC1, APOE, and PVRL2.

Of note, the SNP rs5011804 remains to be significant in the GWAS that correct for APOE $\epsilon 4$ dosage as a covariate; this confirms that the novel SNPs effect is independent from those of APOE $\epsilon 4$ allele. All summary statistics from our GWAS can be found in the Supplementary Material.

\section{Association of rs5011804 with ADAS13, CDRSB, FS Fusiform, and FAQ}

To confirm the direction of the effect of the novel locus, we examined a selection of the biomarkers that were strongly associated with the SNP. The biomarkers ADAS13, CDRSB, FS Fusiform, and FAQ had the smallest additive $p$-values out of all measured QTs associated with rs5011804, as evidenced by Fig. 2(a-d), and as such were selected for further analysis.

Individuals with data for a specific biomarker were sorted into one of three categories based on the number of ' $C$ ' alleles associated with rs5011804 present $(0,1$, or 2 copies). The average level of each of four biomarkers was found for each of the three separate categories, which was then plotted along with the standard error of the mean in Fig. 2(a-d). To doubly verify the independence of the novel SNPs effects from APOE $\epsilon 4$ dosage, the same procedure was followed except for plotting the genetic dosage of the APOE $\epsilon 4$ per individual versus the level of the phenotypic biomarker in Fig. 2(e-h). Additionally, to account for the violation of normality and variance homogeneity in our data, we have plotted a figure similar to Fig. 2 depicting the median and interquartile range instead of the mean and standard error. This updated figure can be seen as Fig. S3 in the Supplementary Material.

To determine the significance of the difference between pairs of averages (i.e. between the average level of a 


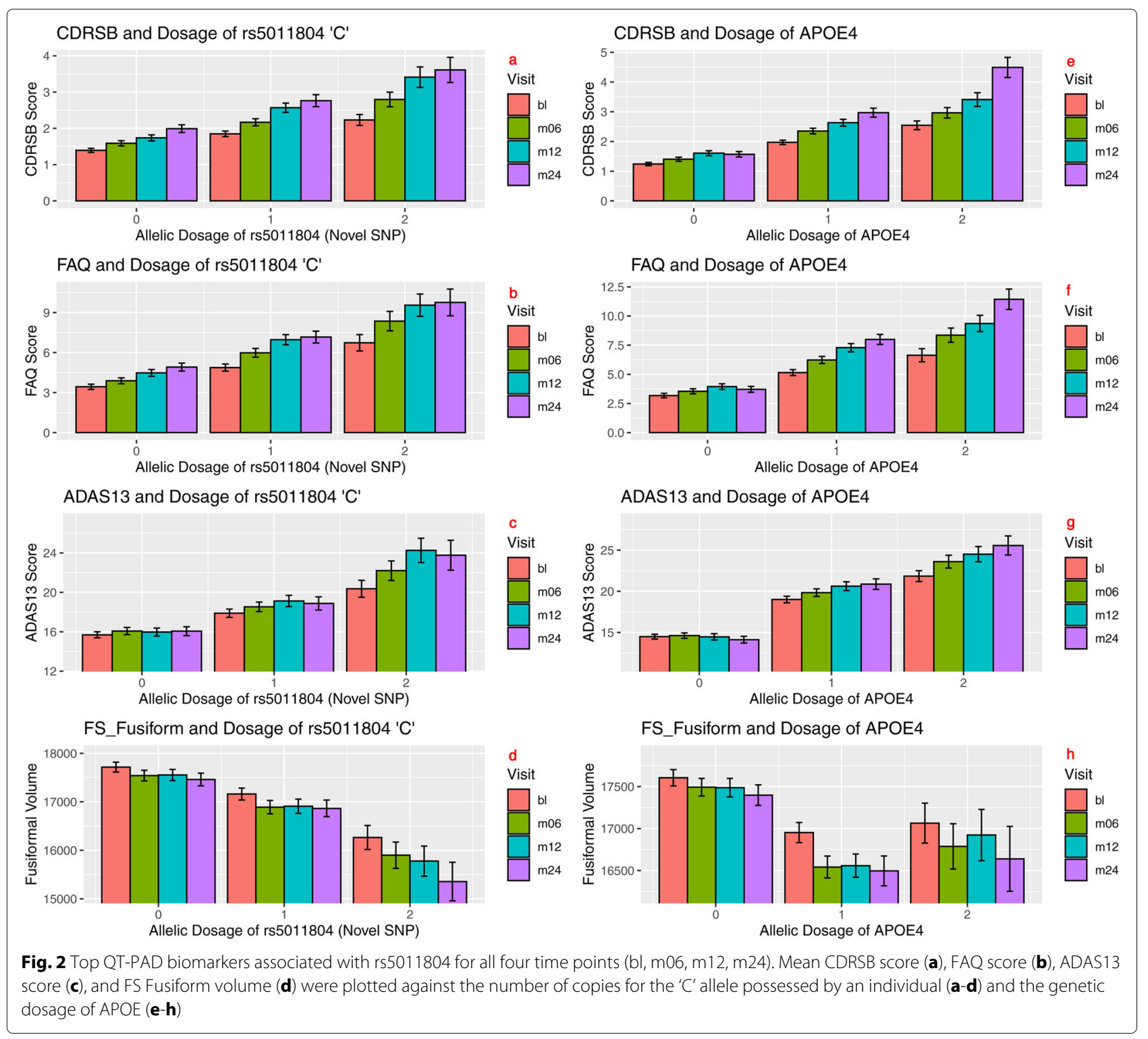

biomarker for individuals with no copies of the novel allele and two copies, individuals with one and two copies, and individuals with no and one copy), Cohen's d values and two-tailed t-test p statistics were computed (Table 1). Similarly, to determine the significance of the difference between pairs of averages (i.e. between the average level of a biomarker for individuals with no copies of the APOE $\epsilon 4$ and two copies, individuals with one and two copies, and individuals with no and one copy), Cohen's $d$ values and two-tailed t-test p statistics were found (Table 2).

The moderately high Cohen's d values and relatively low two-tailed t test $p$ statistics found, especially between individuals with no and two copies of the relevant minor ' $\mathrm{C}$ ' allele, confirm the significant effect this SNP has. From these visualizations, it is apparent that the rs5011804 ' $\mathrm{C}$ ' allele is associated with higher CDRSB, ADAS13, and FAQ scores, which are indicative of a significant cognitive impairment commonly seen in individuals with $\mathrm{AD}$. The same allele is also associated with significantly lower values of the FS Fusiform biomarker, which is consistent with the atrophy expected in neurodegenerative diseases like $\mathrm{AD}$.

In addition to evaluating the effect this SNP has on biomarkers commonly associated with $\mathrm{AD}$, we examined the relationship between the SNP and the diagnosis at each time point. This was done via a case-control linear regression analysis in PLINK v1.90 [37]. Individuals with diagnoses of mild cognitive impairment (MCI) or dementia $(\mathrm{AD})$ were coded as cases while healthy controls $(\mathrm{HC})$ were coded as the controls. The resulting $p$-value 
Table 1 Mann-Whitney U Test $P$ Values for Fig. 2(a-d)

\begin{tabular}{lllll}
\hline Visit (Dosage) & CDRSB P & FAQ P & ADAS13 P & Fusiform P \\
\hline bl (0 vs 1) & $3.59 e-6$ & $1.37 e-5$ & $1.08 \mathrm{e}-4$ & $6.56 \mathrm{e}-2$ \\
bl (0 vs 2) & $2.81 \mathrm{e}-9$ & $1.95 \mathrm{e}-8$ & $3.01 \mathrm{e}-8$ & $4.19 \mathrm{e}-3$ \\
$\mathrm{bl}(1$ vs 2) & $2.22 \mathrm{e}-3$ & $2.63 \mathrm{e}-3$ & $1.29 \mathrm{e}-3$ & $2.47 \mathrm{e}-2$ \\
$\mathrm{~m} 06$ (0 vs 1) & $8.60 \mathrm{e}-6$ & $1.23 \mathrm{e}-6$ & $3.67 \mathrm{e}-5$ & $2.11 \mathrm{e}-1$ \\
$\mathrm{~m} 06$ (0 vs 2) & $1.58 \mathrm{e}-11$ & $1.09 \mathrm{e}-10$ & $2.03 \mathrm{e}-10$ & $4.39 \mathrm{e}-4$ \\
$\mathrm{~m} 06$ (1 vs 2) & $2.44 \mathrm{e}-4$ & $5.69 \mathrm{e}-4$ & $1.56 \mathrm{e}-4$ & $2.37 \mathrm{e}-3$ \\
$\mathrm{~m} 12$ (0 vs 1) & $1.44 \mathrm{e}-7$ & $6.34 \mathrm{e}-7$ & $5.62 \mathrm{e}-6$ & $1.71 \mathrm{e}-1$ \\
$\mathrm{~m} 12$ (0 vs 2) & $3.79 \mathrm{e}-10$ & $7.35 \mathrm{e}-10$ & $1.63 \mathrm{e}-11$ & $7.55 \mathrm{e}-5$ \\
$\mathrm{~m} 12$ (1 vs 2) & $2.15 \mathrm{e}-3$ & $2.30 \mathrm{e}-3$ & $6.14 \mathrm{e}-5$ & $4.19 \mathrm{e}-4$ \\
$\mathrm{~m} 24$ (0 vs 1) & $5.19 \mathrm{e}-4$ & $3.26 \mathrm{e}-4$ & $5.16 \mathrm{e}-3$ & $2.52 \mathrm{e}-1$ \\
$\mathrm{~m} 24$ (0 vs 2) & $7.50 \mathrm{e}-8$ & $1.13 \mathrm{e}-7$ & $3.21 \mathrm{e}-7$ & $5.12 \mathrm{e}-4$ \\
$\mathrm{~m} 24$ (1 vs 2) & $1.29 \mathrm{e}-3$ & $1.87 \mathrm{e}-3$ & $1.03 \mathrm{e}-3$ & $2.94 \mathrm{e}-5$ \\
\hline
\end{tabular}

The index/leftmost column of the table represents the two specific allelic dosage means being compared at a specific time point. For example, in the first row, the differences in the average baseline levels for each of the four listed phenotypes (CDRSB, FAQ, ADAS13, and FreeSurfer Fusiform) are compared between individuals with 0 copies and 1 copy of the minor allele of the SNP rs5011804. The visit codes have been abbreviated as follows: bl represents baseline measures, m06 represents measures at the month 6 visit, $m 12$ represents measures at the month 12 visit, and $\mathrm{m} 24$ represents measures at the month 24 visits. A Mann-Whitney $U$ test $p$ value threshold was determined using the Bonferroni correction, setting a threshold of $1.04 * 10^{-3}$

was $1.47 \times 10^{-3}$, which is significant given a standard Bonferroni-corrected $p$-value threshold of 0.01 .

To confirm the significance of these discrete differences, a chi-squared test were performed for the data at each time point. These tests rejected the null hypothesis that

Table 2 Mann-Whitney U Test $P$ Values for Fig. 2(e-h)

\begin{tabular}{lllll}
\hline Visit (Dosage) & CDRSB P & FAQ P & ADAS13 P & Fusiform P \\
\hline bl (0 vs 1) & $7.20 \mathrm{e}-21$ & $6.65 \mathrm{e}-15$ & $1.26 \mathrm{e}-22$ & $4.08 \mathrm{e}-4$ \\
bl (0 vs 2) & $1.85 \mathrm{e}-23$ & $4.21 \mathrm{e}-15$ & $6.82 \mathrm{e}-25$ & $1.11 \mathrm{e}-2$ \\
bl (1 vs 2) & $5.12 \mathrm{e}-5$ & $2.77 \mathrm{e}-3$ & $1.22 \mathrm{e}-4$ & $4.31 \mathrm{e}-1$ \\
m06 (0 vs 1) & $1.26 \mathrm{e}-20$ & $9.61 \mathrm{e}-16$ & $9.21 \mathrm{e}-20$ & $2.47 \mathrm{e}-6$ \\
m06 (0 vs 2) & $4.70 \mathrm{e}-25$ & $1.84 \mathrm{e}-21$ & $4.15 \mathrm{e}-25$ & $9.68 \mathrm{e}-4$ \\
m06 (1 vs 2) & $3.69 \mathrm{e}-5$ & $3.51 \mathrm{e}-5$ & $3.23 \mathrm{e}-5$ & $1.51 \mathrm{e}-1$ \\
m12 (0 vs 1) & $5.43 \mathrm{e}-17$ & $2.81 \mathrm{e}-17$ & $1.21 \mathrm{e}-12$ & $8.42 \mathrm{e}-4$ \\
m12 (0 vs 2) & $2.97 \mathrm{e}-20$ & $4.69 \mathrm{e}-18$ & $7.29 \mathrm{e}-25$ & $8.60 \mathrm{e}-2$ \\
m12 (1 vs 2) & $1.24 \mathrm{e}-4$ & $9.54 \mathrm{e}-4$ & $6.87-5$ & $1.41 \mathrm{e}-1$ \\
m24 (0 vs 1) & $1.72 \mathrm{e}-16$ & $3.10 \mathrm{e}-18$ & $1.05 \mathrm{e}-20$ & $1.34 \mathrm{e}-3$ \\
m24 (0 vs 2) & $9.06 \mathrm{e}-22$ & $2.10 \mathrm{e}-19$ & $1.98 \mathrm{e}-21$ & $6.57 \mathrm{e}-3$ \\
m24 (1 vs 2) & $8.18 \mathrm{e}-6$ & $2.18 \mathrm{e}-4$ & $6.54 \mathrm{e}-5$ & $3.85 \mathrm{e}-2$
\end{tabular}

The index/leftmost column of the table represents the two specific allelic dosage means being compared at a specific time point. For example, in the first row, the differences in the average baseline levels for each of the four listed phenotypes (CDRSB, FAQ, ADAS13, and FreeSurfer Fusiform) are compared between individuals with 0 copies and 1 copy of the minor allele of the APOE $\epsilon 4$ allele. A Mann-Whitney $\mathrm{U}$ test $p$ value threshold was determined using the Bonferroni correction, setting a threshold of $1.04 * 10^{-3}$ the genetic dosage at each time point is independent from the AD diagnosis with $p<1.00 \times 10^{-5}$ for the bl, m06, and $\mathrm{m} 12$ visit codes and $p=4.77 \times 10^{-4}$ for the $\mathrm{m} 24$ visit code.

\section{Mediation analysis}

Several statistically significant SNP-QT associations (highlighted in Fig. 1(a-d) across all four time points) were found to exhibit a mediation effect. Here the SNP (the independent variable) influences the QT (the mediator variable), which in turn influences the diagnostic outcome (the dependent variable). The proportion of the mediating effect of the QT was calculated and shown in Fig. 3. In this figure, we plot the proportion of the mediation effect calculated against the visit code in question to show the progression of this proportion over time, which both highlights the significance of these effects and confirms the impact of our SNP. We chose to specifically focus on QT outcomes with at least a statistically significant effect at three or more visit codes to ensure significance. Our measurements show that the CDRSB outcome has the largest proportion of the mediating effect. All effects shown and found are consistent with what would be expected in the case of a cognitive dysfunction such as Alzheimer's.

This specific analysis serves to show that the effect this SNP has on an individual's AD diagnosis goes through these significant QTs. The discovered QTs mediate the effects of $\mathrm{AD}$ candidate variants on disease, which may not be directly detected from the SNP-QT association analysis performed earlier. To the best of our knowledge, this is among the first analysis in AD studies to look for QTs mediating genetic effects on AD diagnosis. We have identified multiple QT mediators linking the SNP to the diagnosis, showing the promise this SNP may have a causal mechanism through these QTs to influence AD diagnosis.

\section{Interaction analysis}

Our interaction analysis found seven specific SNP-bydiagnosis interaction relationships of statistical significance determined via a Bonferroni correction. Six cognitive and one imaging QTs exhibited such an effect, as shown in Fig. 4. Among these findings, similar interaction patterns are found on all six cognitive QTs, including FAQ measures (at m06, m12 and m24), CDRSB measures (at m12), MMSE measures (at m12) and ADAS13 measures (at m24). Specifically, for these cognitive QTs, while the SNP rs5011804 demonstrates either an additive effect or no effect in both NL and MCI diagnostic groups, it shows a heterozygous effect in the AD group, where heterozygous $\mathrm{AD}$ patients (i.e., allelic dosage $=1$ ) have the smallest or largest mean FAQ, compared with homozygous AD patients. In contrast, a different interaction pattern is shown on the only imaging QT finding. For 


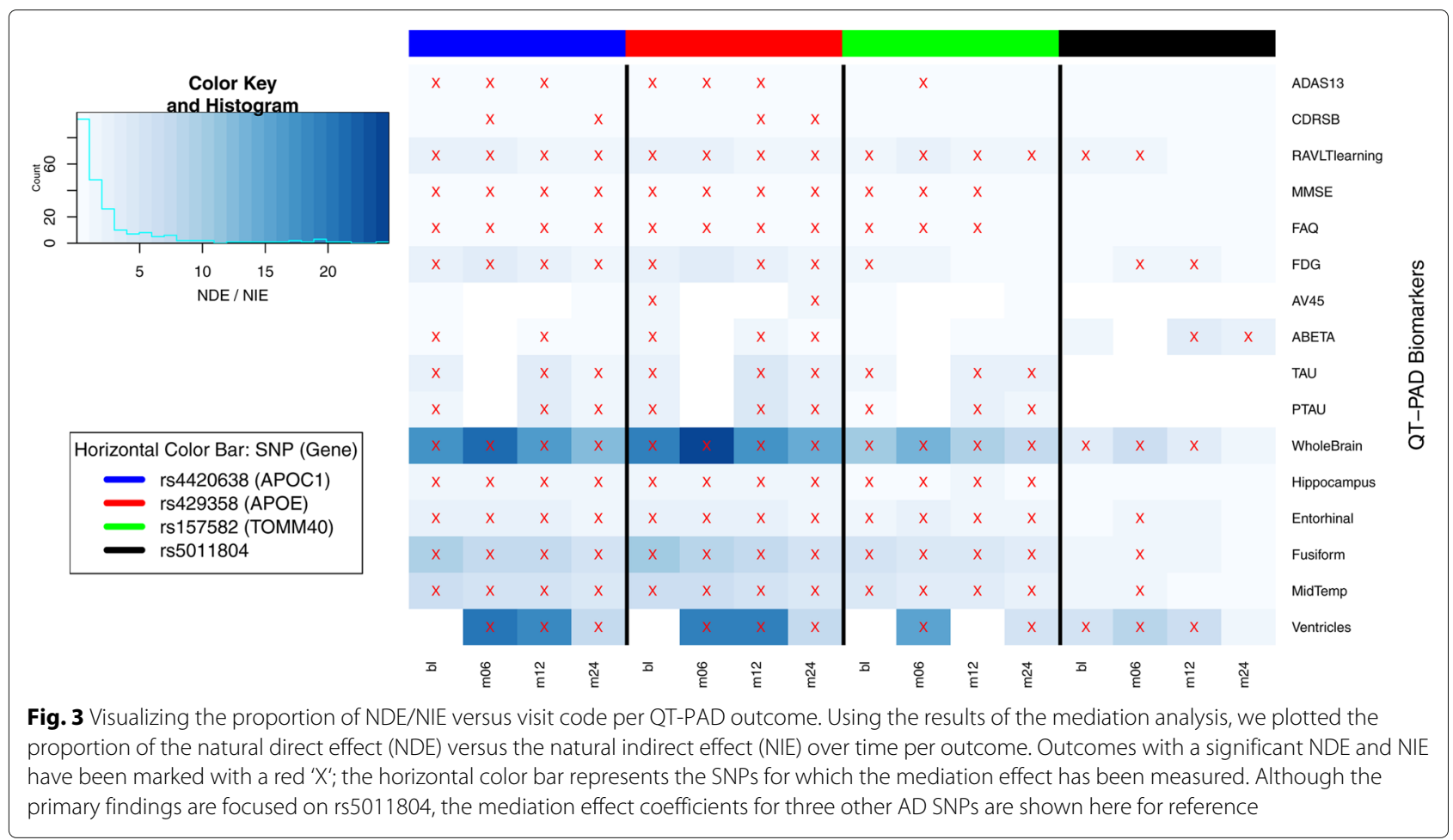

MidTemp measures (at m12), the SNP rs5011804 shows an additive effect in both $\mathrm{MCI}$ and $\mathrm{AD}$ groups, and shows a heterozygous effect in the NL group.

\section{Discussion}

Our GWAS analysis of targeted AD biomarkers discovered a novel SNP rs5011804 at 12p12.1 associated with measures of several quantiative biomarkers in 1,576 members of the QT-PAD cohort. Our post-hoc stratified, mediation, and interaction analyses have yielded a few observations as follows. First, this effect is independent of common AD risk APOE $\epsilon 4$. Second, there is a mediating effect between the SNP and an individual's AD status through a collection of key biomarkers. Finally there exist

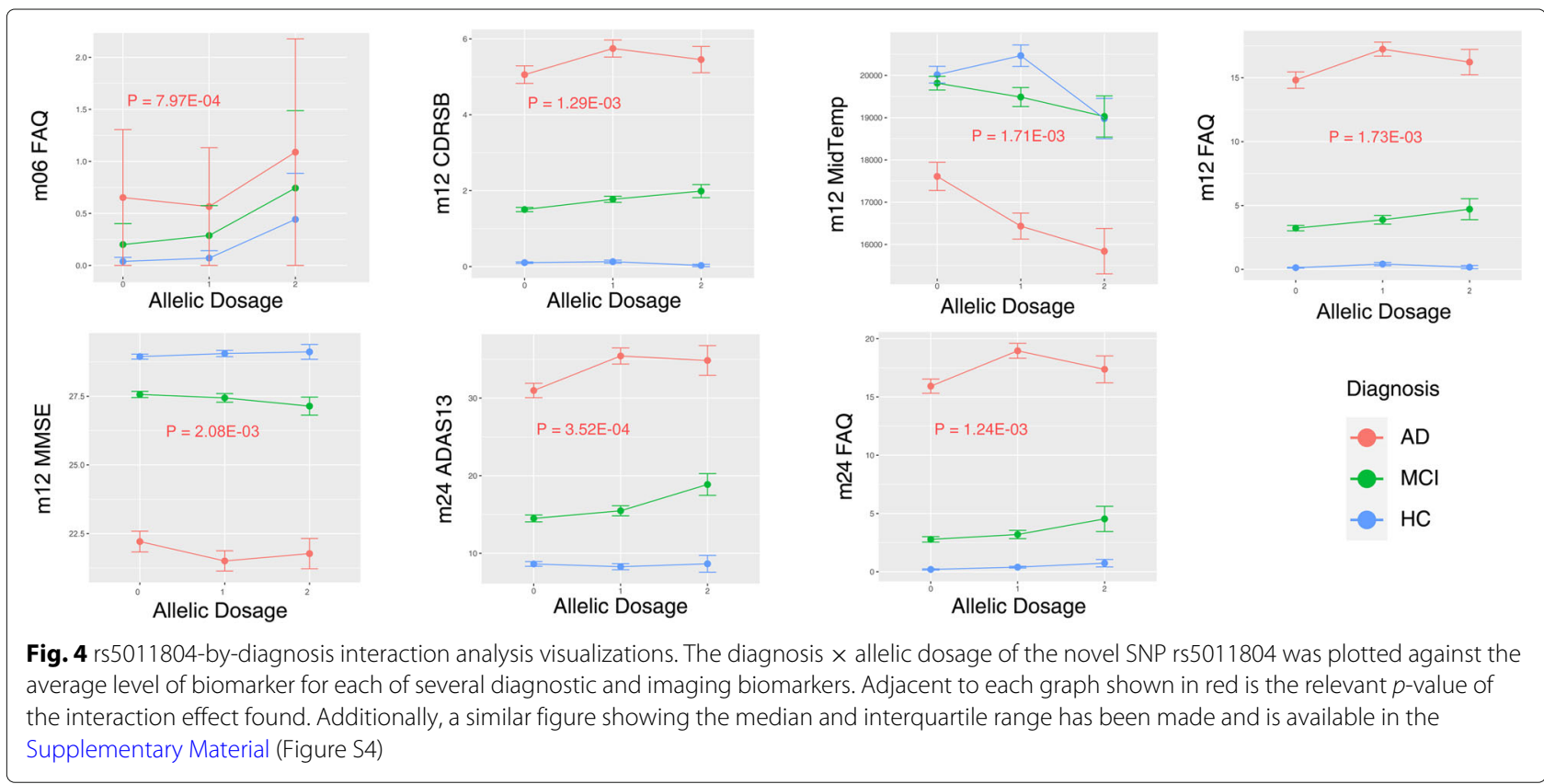


strong SNP-by-diagnosis interaction effects on a few AD biomarkers.

A genome-wide association study has implicated that our novel SNP is highly associated with several AD quantitative traits (QTs) with $p<5.00 \times 10^{-8}$. To our knowledge, this is the first time this SNP has been reported to be strongly associated with AD.

Previously, this SNP has been strongly associated with adult-onset asthma after correcting for smoking habits [38]. The ENIGMA2 project has previously found that rs5011804 has a borderline association with the intercranial volume (ICV) with a $p=0.05934$ [39]. In their late-onset AD GWAS, Kunkle et al. included rs5011804 in their analyses but did not find that it had a statistically significant effect $p=0.7015$ [6].

To better understand the function of the region of chromosome 12 rs5011804 is in, we attempted to find other SNPs in the same LD block. Using the SNPStats R library [40], we searched for all SNPs within a $1 \mathrm{Mb}$ range that were in linkage disequilibrium with rs5011804, as defined by $D^{\prime} \geq 0.8, r^{2} \geq 0.8$, GWAS $p \leq 0.05$. No SNPs were found to be in linkage disequilibrium with rs5011804.

Although rs5011804 is not in linkage disequilibrium with any other currently-recognized SNPs, it is located between genes KRAS (distance $\approx 38 \mathrm{~KB}$ ) and LMNTD1 (distance $\approx 75 \mathrm{~KB}$ ). KRAS is an oncogene that produces K-Ras, a GTPase associated with the RAS/MAPK pathway, which is instrumental in cell growth and differentiation. As such, KRAS has been shown to be associated with disorders including lung cancers and cholangiocarcinoma [41]. LMNTD1, also commonly referred to as PAS1C1, is a protein-encoding gene involved in cell population proliferation that is also associated with lung cancer [42].

To gain more insight into the potential regulatory role of rs5011804, data from the Genotype-Tissue Expression (GTEx) Project was studied. The data analyzed was sourced from the GTEx Portal on 11 November 2020. There were no significant variant-gene associations found in any of the provided brain tissues.

In addition to examining the novel SNP, it is essential to discuss the multiple QTs involved in this study. Multiple QTs associated with the SNP and included in the original QT-PAD dataset are strongly linked with $\mathrm{AD}$, and as such, it both makes sense and is expected that a SNP highly associated with some of these QTs would also have a strong association with $\mathrm{AD}$ directly. For example, the biomarkers ADAS13, FAQ, CDRSB, MMSE, and RAVLT.learning are diagnostic scales commonly used by physicians to quickly assess a patient's mental status and can accurately differentiate between healthy individuals from those with mild cognitive impairments or severe dementia [23-25, 43, 44]. The biomarkers
FDG PET, Amyloid PET, FS WholeBrain, FS Hippocampus, FS Entorhinal, FS Ventricles, FS MidTemp, and FS Fusiform are either molecular imaging measurements or neuroimaging volumetric measurements; previous studies have shown that specific neurological abnormalities can be an effective and accurate way to diagnose a patient with $\mathrm{AD}[26,45,46]$. The final three remaining biomarkers included in QT-PAD - CSF ABETA, CSF TAU, and CSF PTAU - are measurements of $A \beta 42$ and other proteins that are among the best indicators for AD [22]. Given their high association with $\mathrm{AD}$ and quantitative nature, these biomarkers are excellent outcomes variables to use in GWAS.

With the advent of Big Data, genome-wide association studies have quickly became the most commonly accepted method to analyze genetic data in order to learn about the genetic etiology of complex diseases. As helpful and implicating as GWAS may be, however, it is necessary to remember that these studies are purely associative.

As such, it is usually necessary to employ additional procedures to confirm and further elaborate the genetic signal(s) seen in the GWAS.

The use of two post hoc analyses in the case of this study attempts to do so. Specifically, on one hand, we performed mediation analysis and discovered multiple imaging and cognitive traits that mediate the SNP effect on the diagnosis, showing the promise the SNP rs5011804 may have a causal mechanism through these traits to influence $\mathrm{AD}$ diagnosis. On the other hand, we performed a subsequent SNP-by-diagnosis interaction analysis on the studied biomarkers, and revealed several differential SNP-biomarker association patterns in different diagnostic groups. These findings have great potential to help deconvolute mechanistic complexity and better understand disease subtypes.

This study does not claim to prove there is a definite, unambiguous causal relationship between the novel SNP rs5011804 and an AD diagnosis. Determining all of the multiple biological and environmental factors of an $A D$ diagnosis would require several more studies. Although significant in our cohort, it is necessary for this SNP and the QT's studied to be examined in the context of other non-Caucasian and European ethnic groups. Further replication studies can confirm if this SNP, along with some of the implicated neuroimaging biomarkers, could help hint at a potential AD mechanism-of-action worth studying. As the SNP both has a significant effect on AD diagnosis through several AD outcomes measured (seen in the mediation analysis) and exhibits a significant effect on $\mathrm{AD}$ outcomes when combined with an individual's diagnosis (as demonstrated in the interaction analysis), perhaps the SNP may assist in determining an individual's risk for $\mathrm{AD}$ in the future. 


\section{Conclusions}

In conclusion, we discovered a novel locus rs5011804 at 12p12.1 significantly associated with levels of CDRSB, FAQ, FS Fusiform, and ADAS13 at multiple studied time points including the baseline, month 06 , month 12 , and month 24 visits in the ADNI cohort. This locus was also found to be strongly associated with an AD diagnosis. Post hoc mediation and interaction analyses confirmed and elaborated the results of our GWAS. In particular, the genetic effect of this SNP on the AD phenotype is mediated by multiple quantitative biomarkers, suggesting possible causal mechanisms from the SNP to biomarkers and to the diagnostic outcome. In addition, differential SNP-biomarker association patterns are identified in different diagnostic groups, providing valuable information for mechanistic understanding of the disease and heterogeneity. This SNP has never been associated with AD before, and our findings may help lead to a more comprehensive view of the molecular mechanism behind AD.

\section{Methods}

All methods were performed in accordance with the relevant guidelines and regulations. Data were downloaded and analyzed under approval of the University of Pennsylvania Institutional Review Board. An overview of the procedure for this study is shown in Fig. 5. Briefly, after identifying four specific time points to examine data for, multiple GWAS were performed using 1,576 individuals from the QT-PAD cohort and using 16 QTs included in QT-PAD. We performed two sets of these GWAS: one set included APOE $\epsilon 4$, age, gender, and education as covariates; and the other included just age, gender, and education as covariates. After these GWAS, we also performed mediation analysis between the SNP and diagnosis using a QT-PAD biomarker as the mediator variable, and interaction analysis measuring the SNP-by-diagnosis interaction effect on the QT-PAD biomarkers.

\section{Alzheimer's disease neuroimaging initiative QT-PAD data}

Data used in this analysis was obtained from the Alzheimer's Disease Neuroimaging Initiative (ADNI) database [47]. ADNI was launched in 2003 as a publicprivate partnership led by Principal Investigator Michael W. Weiner, MD to test whether serial MRI, PET, and biological markers can be combined with clinical and neuropsychological assessments to accurately measure the progression of mild cognitive impairment (MCI) and early AD. For up-to-date information, see http://www.adniinfo.org.

Participants included individuals who were members of ADNI $1 / \mathrm{GO} / 2$ cohorts, as described by the ADNI QTPAD project (Fig. 5 Box (a)). Please refer to [48] for details about the QT-PAD data and how participants were chosen. Table 3 shows the 16 AD outcomes included in the QT-PAD. To reduce the likelihood of population stratification effects, only non-Hispanic Caucasian participants were involved in this study. As such, there were 1,576 individuals who were studied in each of the four time points. 461 of these individuals are healthy controls (HC) and the remaining 1,115 individuals had either an $\mathrm{MCI}$ or AD diagnosis. Demographic data about the individuals included in our analyses can be found in Table 4.

Genotyping data (Fig. 5 Box (b)) were qualitycontrolled, imputed using 1000G data, and combined as described in $[49,50]$. Briefly, genotyping was performed on all ADNI participants following the manufacturer's protocol using blood genomic DNA samples and Illumina GWAS arrays (610-Quad, OmniExpress, or HumanOmni2.5-4v1) [51]. Quality control was performed in PLINK v1.90 [37] using the following criteria: 1) call

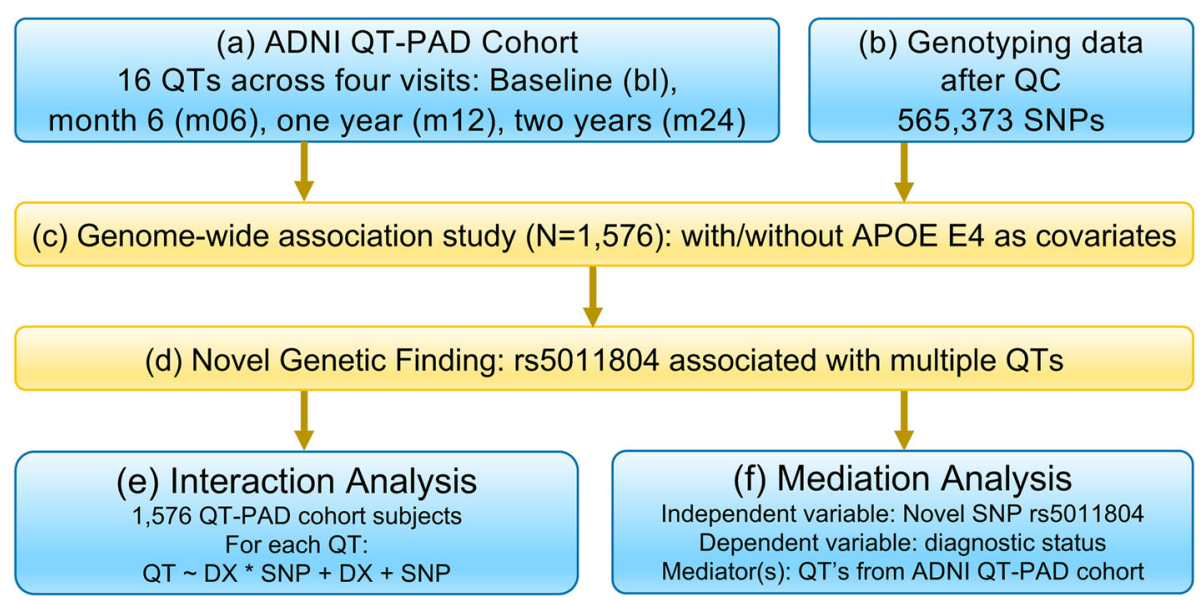

Fig. 5 Workflow. A schematic workflow of the analyses performed in this study 
Table 3 Description of QT-PAD outcomes/biomarkers, their abbreviations, and categories

\begin{tabular}{|c|c|c|}
\hline Abbreviation & Outcome/Biomarker Description & Category \\
\hline ADAS13 & $\begin{array}{l}\text { Alzheimer's Disease Assessment } \\
\text { Scale - Cog } 13\end{array}$ & Cognition \\
\hline CDRSB & $\begin{array}{l}\text { Clinical Dementia Rating - Sum of } \\
\text { Boxes }\end{array}$ & Cognition \\
\hline RAVLT.learning & Rey Auditory Verbal Learning Test & Cognition \\
\hline MMSE & $\begin{array}{l}\text { Mini-Mental State } \\
\text { Examination/Folstein Test }\end{array}$ & Cognition \\
\hline FAQ & Functional Activities Questionnaire & Cognition \\
\hline FDG PET & $\begin{array}{l}\text { Fluorodeoxyglucose Positron } \\
\text { Emission Tomography }\end{array}$ & PET \\
\hline Amyloid PET/AV45 & $\begin{array}{l}\text { Amyloid Positron Emission } \\
\text { Tomography }\end{array}$ & PET \\
\hline CSF ABETA & $\begin{array}{l}\text { Cerebrospinal Fluid Beta-Amyloid } \\
42\end{array}$ & CSF \\
\hline CSFTAU & Cerebrospinal Fluid Tau & CSF \\
\hline CSF PTAU & $\begin{array}{l}\text { Cerebrospinal Fluid } \\
\text { Phosphorylated Tau }\end{array}$ & CSF \\
\hline FS WholeBrain & FreeSurfer Whole Brain Volume & MRI \\
\hline FS Entorhinal & $\begin{array}{l}\text { FreeSurfer Entorhinal Cortex } \\
\text { Volume }\end{array}$ & MRI \\
\hline FS Ventricles & FreeSurfer Ventricular Volume & MRI \\
\hline FS MidTemp & $\begin{array}{l}\text { FreeSurfer Middle Temporal Gyrus } \\
\text { Volume }\end{array}$ & MRI \\
\hline FS Fusiform & FreeSurfer Fusiform Volume & MRI \\
\hline FS Hippocampus & FreeSurfer Hippocampus Volume & MRI \\
\hline
\end{tabular}

rate per marker $\geq 95 \%, 2)$ minor allele frequency $(\mathrm{MAF}) \geq$ $5 \%, 3)$ Hardy Weinberg Equilibrium (HWE) test $\mathrm{P} \leq 1.0 \mathrm{E}-$ 6 , and 4) call rate per participant $\geq 95 \%$. In this study, we analyzed the genetic markers available on the ADNI1610 -Quad panel, where a total of 565,373 SNPs were included in the GWAS.

Table 4 ADNI QT-PAD Participant Characteristics. Gender, age (in years), education (in years), and genetic dosage of the $\mathrm{APOE} \epsilon 4$ allele at the baseline are shown

\begin{tabular}{|c|c|c|c|c|}
\hline Diagnosis & $\mathrm{HC}$ & $\mathrm{MCl}$ & $A D$ & N/A \\
\hline Number & 461 & 797 & 312 & 6 \\
\hline $\begin{array}{l}\text { Gender (\% } \\
\text { Female) }\end{array}$ & 49.67 & 40.15 & 42.95 & 66.67 \\
\hline Age (Mean \pm std) & $74.47 \pm 5.68$ & $73.09 \pm 7.54$ & $75.18 \pm 7.79$ & $72.15 \pm 8.29$ \\
\hline $\begin{array}{l}\text { Education } \\
\text { (Meantstd) }\end{array}$ & $16.42 \pm 2.65$ & $16.00 \pm 2.82$ & $15.24 \pm 2.96$ & $16.75 \pm 1.50$ \\
\hline $\begin{array}{l}\text { APOE } \epsilon 4 \text { Dosage } \\
(\text { Mean } \pm s t d)\end{array}$ & $0.31 \pm 0.51$ & $0.62 \pm 0.68$ & $0.85 \pm 0.71$ & $0.25 \pm 0.50$ \\
\hline
\end{tabular}

Individuals have been sorted into strata depending on their diagnosis at the baseline visit: healthy control (HC), mild cognitive impairment (MCl), Alzheimer's Disease (AD), or not applicable for individuals who do not have baseline diagnosis data enclosed (N/A). The mean and standard deviation (std) are provided for the quantitative measures

\section{Genome wide association studies}

To analyze data from multiple time points, multiple GWAS (Fig. 5 Box (c)) were performed, with one analysis per each of the four time points bl, $\mathrm{m} 06, \mathrm{~m} 12$, and $\mathrm{m} 24$, which represented the baseline, month 6 , month 12 , and month 24 visits. Due to the large extent of ADNI as a whole and the difficulties each individual patient might have had, not every patient has a recorded value in QTPAD stored for each of the 16 biomarkers at each visit. To ensure our analyses to have enough statistical power, we only studied time points with a minimum of 200 individuals. As such, our analysis was limited to the four aforementioned time points.

Targeted genetic association analysis of each of the 16 AD biomarkers at each of the four listed time points on the 565,373 SNPs was tested using linear regression under an additive genetic model in PLINK v1.90 [37]. Initially, age, gender, and education only were used as covariates in our GWAS. To correct for the effects of APOE $\epsilon 4$ status (best known $\mathrm{AD}$ genetic risk factor), GWAS was also performed using the exact same data with age, gender, education, and APOE $\epsilon 4$ dosage as covariates. For both trials, significant SNP-QT associations were reported using the genome-wide significance threshold of $p \leq 5.00 \times 10^{-8}$.

\section{Mediation analysis}

Given the dynamic nature of Alzheimer's disease, a cohort may have a varying distribution along the $\mathrm{HC}-\mathrm{MCI}-\mathrm{AD}$ spectrum as seen through varying biomarker levels and changing diagnoses. In the context of a GWAS, having a dynamic phenotype but a static genetic basis over time can seem contradictory, allowing for the possibility of certain genetic factors being significantly associated with an $\mathrm{AD}$ diagnosis or biomarkers closely linked to $\mathrm{AD}$ at one time point but not another. In order to accommodate such an effect and verify the highlighted SNP indeed plays a role in $\mathrm{AD}$ diagnosis and outcomes at all significant time points, we propose a mediation analysis.

A mediation analysis seeks to identify and explain the mechanism of the quantified relationship between rs5011804 and an $\mathrm{AD}$ diagnosis via examining the mediating effects of the various ADNI QT-PAD biomarkers discussed (see Fig. 6). Specifically, a mediation analysis will allow us to determine if an independent variable (the SNP rs5011804) affects a dependent variable (an individual's AD diagnosis) 'through' one of mediator variables (our biologically-motivated AD outcomes). Since many of these QT-PAD outcomes and biomarkers have distinct biological ties to the disease itself, a mediation analysis would both hint at a causal relationship between SNP and diagnosis as well as hint at a possible mechanism of action, drug target, or region of interest (ROI). Below we summarize the specific mediation analysis performed (Fig. 5 Box (f)). For each time point, we followed [52] to perform 


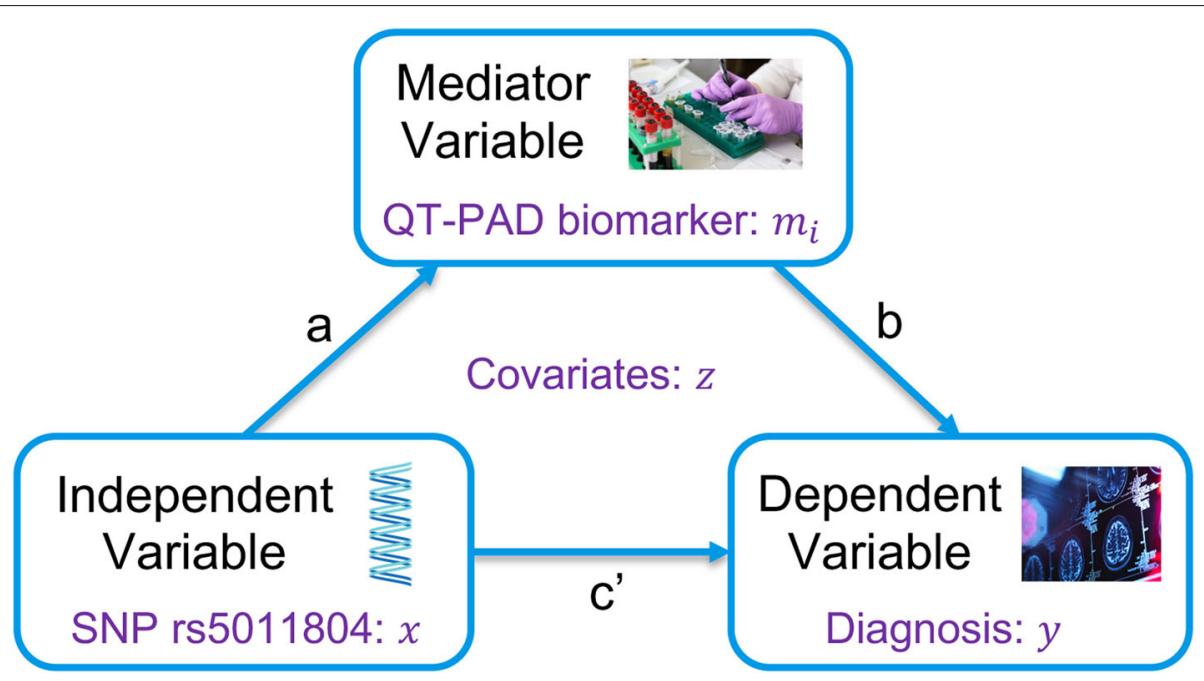

Fig. 6 Mediation analysis. Our mediation analysis aims to determine if an independent variable (SNP rs5011804) affects a dependent variable (diagnosis) 'through' a mediator variable (QT-PAD biomarker), where age, gender and education are included as covariates. The direct effect is the path coefficient $c^{\prime}$. The indirect effect is the path coefficent product $a \times b$

a standard mediation analysis to identify key biomarkers included in QT-PAD as potential disease moderators. Figure 6 shows a brief graphical summary of this method.

Let $y \in\{1,2\}$ be the dependent variable which represents the diagnostic phenotype in the study, with 2 representing a case (diagnosis of either $\mathrm{MCI}$ or $\mathrm{AD}$ ) and 1 representing a healthy control; $x \in\{0,1,2\}$ be the independent variable which represents the allelic dosage of the minor allele ' $\mathrm{C}$ ' in our identified SNP rs5011804, with 2 signifying an individual has two copies of the minor allele, 1 signifying an individual has one copy of the SNP, and 0 signifying the individual does not carry the variation; $\mathrm{z}$ be the covariates (age, gender, and education but not APOE $\epsilon 4$ dosage); and $M$ be the set of significant biomarkers at each of the four time points as indicated by the previous GWAS. Mediation analysis was performed via the three steps below:

Step 1: We use a logistic regression model to regress an individual's diagnosis $y$ against the SNP $x$ while controlling for the covariates $z$.

$$
\operatorname{logit}(\operatorname{Pr}(y=2))=\beta_{11} x+\beta_{12} z+\epsilon_{1}
$$

Coefficient $\beta_{11}$ should be significant $(p$-value $<0.05)$ to pass this first step.

Step 2: We use a linear regression model to regress each of the potentially mediating biomarkers denoted $m_{i}$ (i.e., $m_{i} \in M$ ) against the SNP $x$ while controlling for the covariates $z$.

$$
m_{i}=\beta_{21, i} x+\beta_{22, i} z+\epsilon_{2, i}
$$

We only use the SNP rs5011804 - and therefore continue with this post hoc analysis - if it meets the significance threshold of 0.05 . Coefficient $\beta_{21, i}$ should be significant after correcting for the multiple biomarkers; we correct our $p$-value threshold using a Bonferroni correction. As such, given that the number of biomarkers at each step differ, we have a threshold of $\frac{0.05}{7}=7.14 \times 10^{-3}$ for the baseline data, a threshold of $\frac{0.05}{6}=8.33 \times 10^{-3}$ for the $\mathrm{m} 06$ and $\mathrm{m} 12$ data, and a threshold of $\frac{0.05}{4}=1.25 \times 10^{-2}$ for the m24 data.

Step 3: We use a logistic regression model to regress an individual's diagnosis $y$ against the SNP $x$ and each mediating biomarker phenotype $m_{i}$, controlling for the covariates $z$.

$$
\operatorname{logit}(\operatorname{Pr}(y=2))=\beta_{31, i} x+\beta_{32, i} m_{i}+\beta_{33, i} z+\epsilon_{3, i}
$$

Note that this step is only performed using all mediating phenotypes that satisfy the conditions of the previous step. To adjust for multiple comparisons, we again employ the Bonferroni correction to our significance threshold using the number of mediators surviving the previous step. For any given mediating biomarker $m_{i}$, there is likely a mediating relationship if:

(1) $\beta_{32, i}$ is statistically significant as deemed by our Bonferroni-corrected threshold

(2) $\left|\beta_{31, i}\right|<\left|\beta_{11}\right|$ (from Step 1, above). In other words, an indirect effect must be present between our dependent variable and our independent variable through our mediator.

Next, it is possible to compare the multiple mediation effects we have isolated as introduced in [53]; see also Fig. 6. We have also calculated the proportion of the Natural Direct Effect (NDE), which is expressed as $\beta_{31, i}$, to the Natural Indirect Effect (NIE) $\beta_{32, i} \times \beta_{21, i}$. 
This analysis was performed for all significant SNP-QT associations from our GWAS.

\section{Interaction analysis}

Lastly, in addition to examining the main effect of the SNP on the 16 QT-PAD outcomes, we also perform a SNP-by-diagnosis analysis on these QTs (denoted $L_{Q T}$ ). We primarily consider the novel SNP rs5011804, modeling its allelic effect $x_{a}$ by coding the genotypes as $x_{a}=$ $0,1,2$. Similarly, we code an individual's diagnosis as $x_{d}=$ $0,1,2$, with $\mathrm{HC}$ individuals being coded as 0 , individuals diagnosed with $\mathrm{MCI}$ as 1 , and individuals with an $\mathrm{AD}$ diagnosis being coded as 2 . By multiplication, we obtain the interaction term $x_{a} x_{d}$ which represents interaction between the allelic effect of the SNP and an individual's diagnosis.

As such, we employ the following model to measure the interaction effect $x_{a} x_{d}$ while also controlling for age $\left(c_{\text {age }}\right)$, gender $\left(c_{g e n}\right)$, and education $\left(c_{e d u}\right)$.

$$
L_{Q T}=\beta_{0}+\beta_{1} x_{a}+\beta_{2} x_{d}+\beta_{\text {int }} x_{a} x_{d}+c_{\text {age }}+c_{\text {gen }}+c_{\text {edu }}
$$

We wish to determine the significance of $\beta_{\text {int }}$. Given that we are building multiple models, we use a Bonferroni correction to filter out false positives. As we examine QTs from each time point separately, the Bonferroni threshold is calculated as 0.05 divided by the number of statistically significant QTs in a specific visit as determined by the calculations above. Inspiration for this model was taken from [54].

\begin{abstract}
Abbreviations
AD: Alzheimer's Disease; ADNI: Alzheimer's Disease Neuroimaging Initiative; GWAS: genome-wide association study; SNPs: single nucleotide polymorphisms; QT: quantitative trait(s); CDRSB: Clinical Dementia Rating Sum of Boxes; FAQ: Functional Activities Questionnaire; ADAS13: Alzheimer's Disease Assessment Scale 13-item Cognitive Subscale; FS: FreeSurfer (software); QT-PAD: Quantitative Template for the Progression of Alzheimer's Disease; CSF: cerebrospinal fluid; HC: healthy control; $\mathrm{MCl}$ : mild cognitive impairment; bl: baseline visit; m06: month 6 visit; m12: month 12 visit; m24: month 24 visit; ENIGMA2: Enhancing Neuro Imaging Genetics Through Meta Analysis (Second Continuation); ICV: intercranial volume; LD: linkage disequilibrium; Mb: megabase(s); GTEx: Genotype-Tissue Expression (Project)
\end{abstract}

\section{Supplementary Information}

The online version contains supplementary material available at https://doi.org/10.1186/s12864-021-08269-8.

Additional file 1: Supplementary Material.

\section{Acknowledgements}

Data collection and sharing for this project was funded by the Alzheimer's Disease Neuroimaging Initiative (ADNI) (National Institutes of Health Grant U01 AG024904) and DOD ADNI (Department of Defense award number W81XWH-12-2-0012). ADNI is funded by the National Institute on Aging, the National Institute of Biomedical Imaging andBioengineering, and through generous contributions from the following: AbbVie, Alzheimer's Association; Alzheimer's Drug Discovery Foundation; Araclon Biotech; BioClinica, Inc:; Biogen; Bristol-Myers Squibb Company; CereSpir, Inc.; Cogstate; Eisai Inc.; Elan Pharmaceuticals, Inc.; Eli Lilly and Company; Eurolmmun; F. Hoffmann-La Roche Ltd and its affiliated company Genentech, Inc.; Fujirebio; GE Healthcare;
IXICO Ltd.; Janssen Alzheimer Immunotherapy Research \& Development, LLC.; Johnson \& Johnson Pharmaceutical Research \& Development LLC.; Lumosity; Lundbeck; Merck \& Co., Inc.; Meso Scale Diagnostics, LLC.; NeuroRx Research; Neurotrack Technologies; Novartis Pharmaceuticals Corporation; Pfizer Inc.; Piramal Imaging; Servier; Takeda Pharmaceutical Company; and Transition Therapeutics. The Canadian Institutes of Health Research is providing funds to support ADNI clinical sites in Canada. Private sector contributions are facilitated by the Foundation for the National Institutes of Health (www.fnih.org). The grantee organization is the Northern California Institute for Research and Education, and the study is coordinated by the Alzheimer's Therapeutic Research Institute at the University of Southern California. ADNI data are disseminated by the Laboratory for Neuro Imaging at the University of Southern California.

Data used in preparation of this article were obtained from the Alzheimer's Disease Neuroimaging Initiative (ADNI) database (adni.loni.usc.edu). As such, the investigators within the ADNI contributed to the design and implementation of $\mathrm{ADNI}$ and/or provided data, but did not participate in analysis or writing of this report. A complete listing of ADNI investigators can be found at: http://adni.loni.usc.edu/wp-content/uploads/how_to_apply/ ADNI_Acknowledgement_List.pdf.

\section{Authors' contributions}

$L S$ and $B L$ designed the study. XY performed genotyping data preparation. Method implementation, data analysis and result interpretation were performed by $B L$, guided by $L S$, and assisted by $X Y$. The initial document was drafted by BL and LS. All the authors reviewed, commented, revised and approved the manuscript.

\section{Funding}

This work and publication costs were supported by National Institute of Health U01 AG068057, R01 AG071470, RF1 AG068191, and R01 LM013463.

\section{Availability of data and materials}

Data used in the preparation of this article were downloaded from the Alzheimer's Disease Neuroimaging Initiative (ADNI) database. The ADNI data are available to the public at http://adni.loni.usc.edu/ through completion of an online application form and acceptance of Data Use Agreement.

\section{Declarations}

Ethics approval and consent to participate

Data used in the preparation of this article are the existing data available at the ADNI website (http://adni.loni.usc.edu/), and all the data are not identifiable. Data were downloaded and analyzed under approval of the University of Pennsylvania Institutional Review Board.

\section{Consent for publication}

Not applicable.

\section{Competing interests}

The authors declare that they have no competing interests.

\section{Author details}

${ }^{1}$ Department of Biostatistics, Epidemiology and Informatics, Perelman School of Medicine at the University of Pennsylvania, Philadelphia, USA. ${ }^{2}$ Alzheimer's Disease Neuroimaging Initiative Informatics Core, Los Angeles, USA.

Received: 5 June 2021 Accepted: 14 December 2021

Published online: 28 January 2022

\section{References}

1. Dubois B, Hampel H, et al. Preclinical Alzheimer's disease: Definition, natural history, and diagnostic criteria. 2016;12(3):292-323. https://doi. org/10.1016/j.jalz.2016.02.002.

2. King A. The search for better animal models of Alzheimer's disease. Nature. 2018;559(7715):13-5. https://doi.org/10.1038/d41586-018-05722-9.

3. Van Cauwenberghe C, Van Broeckhoven C, Sleegers K. The genetic landscape of Alzheimer disease: clinical implications and perspectives. Genet Med. 2016;18(5):421-30. https://doi.org/10.1038/gim.2015.117. 
4. Gatz M, Reynolds CA, et al. Role of genes and environments for explaining Alzheimer disease. Arch Gen Psychiatry. 2006;63(2):168-74. https://doi.org/10.1001/archpsyc.63.2.168.

5. Roussotte FF, Daianu M, et al. Neuroimaging and genetic risk for alzheimer's disease and addiction-related degenerative brain disorders. Brain Imaging Behav. 2014;8(2):217-33.

6. Kunkle BW, Grenier-Boley B, et al. Genetic meta-analysis of diagnosed alzheimer's disease identifies new risk loci and implicates abeta, tau, immunity and lipid processing. Nat Genet. 2019;51(3):414-30.

7. Jansen IE, Savage JE, et al. Genome-wide meta-analysis identifies new loci and functional pathways influencing alzheimer's disease risk. Nat Genet. 2019;51(3):404-13.

8. Lambert JC, Ibrahim-Verbaas CA, et al. Meta-analysis of 74,046 individuals identifies 11 new susceptibility loci for alzheimer's disease. Nat Genet. 2013;45(12):1452-8

9. Weiner MW, Veitch DP, Aisen PS, Beckett LA, Cairns NJ, Green RC, Harvey D, Jack CR, Jagust W, Liu E, Morris JC, Petersen RC, Saykin AJ, Schmidt ME, Shaw L, Shen L, Siuciak JA, Soares H, Toga AW, Trojanowski JQ, Alzheimer's Disease Neuroimaging Initiative. The Alzheimer's Disease Neuroimaging Initiative: a review of papers published since its inception. Alzheimers Dement. 2013;9(5):111-94. https://doi.org/10.1016/j.jalz.2013.05.1769.

10. Weiner MW, Veitch DP, et al. The Alzheimer's Disease Neuroimaging Initiative 3: continued innovation for clinical trial improvement. Alzheimers Dement. 2017;13(5):561-71. https://doi.org/10.1016/j.jalz.2016.10.006.

11. Weiner MW, Veitch DP, et al. Recent publications from the Alzheimer's Disease Neuroimaging Initiative: reviewing progress toward improved ad clinical trials. Alzheimers Dement. 2017;13(4):1-85. https://doi.org/10. 1016/j.jalz.2016.11.007.

12. Shen $L$, Thompson PM. Brain imaging genomics: Integrated analysis and machine learning. Proc IEEE Inst Electr Electron Eng. 2020;108(1):125-62. https://doi.org/10.1109/JPROC.2019.2947272.

13. Shen $L$, Thompson PM, et al. Genetic analysis of quantitative phenotypes in $\mathrm{AD}$ and $\mathrm{MCl}$ : Imaging, cognition and biomarkers. Brain Imaging Behav. 2014;8(2):183-207. https://doi.org/10.1007/s11682-013-9262-z.

14. Saykin AJ, Shen L, et al. Genetic studies of quantitative $\mathrm{MCl}$ and $A D$ phenotypes in ADNI: Progress, opportunities, and plans. 2015;11(7): 792-814. https://doi.org/10.1016/j.jalz.2015.05.009.

15. Cong S, Yao X, Huang Z, Risacher SL, Nho K, Saykin AJ, Shen L, Consortium UKBE, Initiative ADN. Volumetric gwas of medial temporal lobe structures identifies an erc1 locus using adni high-resolution t2-weighted mri data. Neurobiol Aging. 2020;95:81-93.

16. Ramanan VK, Risacher SL, Nho K, Kim S, Swaminathan S, Shen L, Foroud TM, Hakonarson H, Huentelman MJ, Aisen PS, Petersen RC, Green RC, Jack CR, Koeppe RA, Jagust WJ, Weiner MW, Saykin AJ, Alzheimer's Disease Neuroimaging Initiative. Apoe and bche as modulators of cerebral amyloid deposition: a florbetapir pet genome-wide association study. Mol Psychiatry. 2014;19(3):351-7. https://doi.org/10.1038/mp.2013.19.

17. Yao X, Cong S, Yan J, Risacher SL, Saykin AJ, Moore JH, Shen L, Consortium UKBE, Alzheimer's Disease Neuroimaging I. Regional imaging genetic enrichment analysis. Bioinformatics. 2020;36(8):2554-60.

18. Yao X, Risacher SL, Nho K, Saykin AJ, Wang Z, Shen L, Alzheimer's Disease Neuroimaging, Initiative. Targeted genetic analysis of cerebral blood flow imaging phenotypes implicates the inpp5d gene. Neurobiol Aging. 2019;81:213-21. https://doi.org/10.1016/j.neurobiolaging.2019.06.003.

19. Ramanan VK, Risacher SL, et al. Gwas of longitudinal amyloid accumulation on 18f-florbetapir pet in Alzheimer's disease implicates microglial activation gene il1rap. Brain. 2015;138(10):3076-88. https://doi. org/10.1093/brain/awv231.

20. Agora by NIA AMP-AD Consortium. Nominated Target List (for new Alzheimer's Disease treatment or prevention). 2019. https://agora. ampadportal.org/genes/(genes-router:genes-list). Accessed 5 June 2021.

21. The MODEL-AD Consortium. Jax stock \#003284: II-1r acp ko mouse strain. 2019. https://www.jax.org/strain/003284. Accessed 5 June 2021.

22. Jedynak BM, Lang A, Liu B, et al. A computational neurodegenerative disease progression score: Method and results with the Alzheimer's disease neuroimaging initiative cohort. Neurolmage. 2012;63(3):1478-86. https://doi.org/10.1016/j.neuroimage.2012.07.059.

23. Skinner J, Carvalho JO, Potter GG, et al. The Alzheimer's Disease Assessment Scale-Cognitive-Plus (ADAS-Cog-Plus): an expansion of the
ADAS-Cog to improve responsiveness in $\mathrm{MCl}$. Brain Imaging Behav. 2012;6(4):489-501. https://doi.org/10.1007/s11682-012-9166-3.

24. Estévez-González A, Kulisevsky J, Boltes A, et al. Rey verbal learning test is a useful tool for differential diagnosis in the preclinical phase of Alzheimer's disease: Comparison with mild cognitive impairment and normal aging. Int J Geriatr Psychiatry. 2003;18(11):1021-8. https://doi.org/ 10.1002/gps.1010

25. Trivedi D. Cochrane Review Summary: Mini-Mental State Examination (MMSE) for the detection of dementia in clinically unevaluated people aged 65 and over in community and primary care populations. Prim Health Care Res Dev. 2017;18(6):1-2. https://doi.org/10.1017/ S1463423617000202.

26. Lehmann M, Douiri A, Kim LG, et al. Atrophy patterns in Alzheimer's disease and semantic dementia: A comparison of FreeSurfer and manual volumetric measurements. Neurolmage. 2010;49(3):2264-74. https://doi. org/10.1016/j.neuroimage.2009.10.056.

27. Franke B, van Hulzen KJE, Arias-Vasquez A, et al. Genetic influences on schizophrenia and subcortical brain volumes: Large-scale proof of concept. Nat Neurosci. 2016;19(3):420-31. https://doi.org/10.1038/nn.4228.

28. Deming Y, Li Z, Kapoor M, et al. Genome-wide association study identifies four novel loci associated with Alzheimer's endophenotypes and disease modifiers. Acta Neuropathol. 2017;133(5):839-56. https://doi. org/10.1007/s00401-017-1685-y.

29. Potkin SG, Guffanti G, Lakatos A, et al. Hippocampal Atrophy as a Quantitative Trait in a Genome-Wide Association Study Identifying Novel Susceptibility Genes for Alzheimer's Disease. PLoS ONE. 2009;4(8):6501. https://doi.org/10.1371/journal.pone.0006501.

30. Stein JL, Hua X, Morra JH, et al. Genome-wide analysis reveals novel genes influencing temporal lobe structure with relevance to neurodegeneration in Alzheimer's disease. Neurolmage. 2010;51(2): 542-54. https://doi.org/10.1016/j.neuroimage.2010.02.068.

31. Furney SJ, Simmons A, Breen G, et al. Genome-wide association with MR atrophy measures as a quantitative trait locus for Alzheimer's disease. Mol Psychiatry. 2011;16(11):1130-8. https://doi.org/10.1038/mp.2010.123.

32. Han MR, Schellenberg GD, Wang LS. Genome-wide association reveals genetic effects on human $A \beta 42$ and $\tau$ protein levels in cerebrospinal fluids: A case control study. BMC Neurol. 2010;10:. https://doi.org/10. 1186/1471-2377-10-90

33. Kim S, Swaminathan S, Shen L, et al. Genome-wide association study of CSF biomarkers A $\beta 1-42$, t-tau, and p-tau181p in the ADNI cohort. Neurology. 2011;76(1):69-79. https://doi.org/10.1212/WNL. Ob013e318204a397.

34. Cruchaga C, Kauwe JSK, Harari O, et al. GWAS of cerebrospinal fluid tau levels identifies risk variants for alzheimer's disease. Neuron. 2013;78(2): 256-68. https://doi.org/10.1016/j.neuron.2013.02.026.

35. Keenan BT, Shulman JM, Chibnik LB, et al. A coding variant in CR1 interacts with APOE- $\epsilon 4$ to influence cognitive decline. Hum Mol Genet. 2012;21(10):2377-88. https://doi.org/10.1093/hmg/dds054.

36. Hu X, Pickering EH, Hall SK, Naik S, et al. Genome-wide association study identifies multiple novel loci associated with disease progression in subjects with mild Cognitive impairment. Transl Psychiatry. 2011;1(11):54. https://doi.org/10.1038/tp.2011.50.

37. Purcell S, Neale B, et al. PLINK: A tool set for whole-genome association and population-based linkage analyses. Am J Hum Genet. 2007;81(3): 559-75. https://doi.org/10.1086/519795.

38. Vonk JM, Scholtens $S$, et al. Adult onset asthma and interaction between genes and active tobacco smoking: The GABRIEL consortium. PLoS ONE. 2017;12(3):. https://doi.org/10.1371/journal.pone.0172716.

39. Hibar DP, Stein JL, et al. Common genetic variants influence human subcortical brain structures. Nature. 2015;520(7546):224-9. https://doi. org/10.1038/nature14101.

40. Clayton D. snpStats: SnpMatrix and XSnpMatrix classes and methods. 2020. https://doi.org/10.18129/B9.bioc.snpStats.

41. Addissie YA, Kotecha U, Hart RA, et al. Craniosynostosis and Noonan syndrome with KRAS mutations: Expanding the phenotype with a case report and review of the literature. Am J Med Genet A. 2015;167(11): 2657-63. https://doi.org/10.1002/ajmg.a.37259.

42. Wang $M$, Futamura $M$, Wang $Y$, You M. Pas1C1 is a candidate for the mouse pulmonary adenoma susceptibility 1 locus. Oncogene. 2005;24(11):1958-63. https://doi.org/10.1038/sj.onc.1208295. 
43. Morris JC. The clinical dementia rating (cdr): Current version and scoring rules. Neurology. 1993;43(11):2412-4. https://doi.org/10.1212/wnl.43.11. 2412-a.

44. RI P, TTK, CH H, JM C, S F. Measurement of functional activities in older adults in the community. J Gerontol. 1982;37(3). https://doi.org/10.1093/ GERONJ/37.3.323.

45. Suppiah S, Didier MA, Vinjamuri S. The who, when, why, and how of PET amyloid imaging in management of Alzheimer's disease-review of literature and interesting images. MDPI AG. 2019. https://doi.org/10.3390/ diagnostics9020065.

46. Ou YN, Xu W, Li JQ, et al. FDG-PET as an independent biomarker for Alzheimer's biological diagnosis: A longitudinal study. Alzheimers Res Ther. 2019;1 1(1):57. https://doi.org/10.1186/s13195-019-0512-1.

47. ADNI. Alzheimer's Disease Neuroimaging Initiative. 2012. http://adni.loni. usc.edu/. Accessed 15 June 2020.

48. Portland Institute for Computational Science. Alzheimer's Disease Modelling Challenge: Modelling the progression of Alzheimer's disease. 2012. http://www.pi4cs.org/qt-pad-challenge. Accessed 15 June 2020.

49. Yao X, Cong $\mathrm{S}$, et al. Regional imaging genetic enrichment analysis. Bioinformatics. 2019;36(8):2554-60.

50. Yao X, Risacher SL, et al. Targeted genetic analysis of cerebral blood flow imaging phenotypes implicates the inpp5d gene. Neurobiol Aging. 2019;81:213-21.

51. Saykin AJ, Shen $L$, et al. Alzheimer's disease neuroimaging initiative biomarkers as quantitative phenotypes: Genetics core aims, progress, and plans. Alzheimers Dement. 2010;6(3):265-73.

52. Baron RM, Kenny DA. The Moderator-Mediator Variable Distinction in Social Psychological Research: Conceptual, Strategic, and Statistical Considerations. J Personal Soc Psychol. 1986;51(6):1173-82.

53. Breen R, Karlson KB, Holm A. Total, Direct, and Indirect Effects in Logit and Probit Models. Sociol Methods Res. 2016;42(2):164-91. https://doi. org/10.1177/0049124113494572.

54. Herold C, Steffens M, Brockschmidt FF, Baur MP, Becker T. INTERSNP: genome-wide interaction analysis guided by a priori information. Bioinformatics. 2009;25(24):3275-81. https://doi.org/10.1093/ bioinformatics/btp596.

\section{Publisher's Note}

Springer Nature remains neutral with regard to jurisdictional claims in published maps and institutional affiliations.

Ready to submit your research? Choose BMC and benefit from:

- fast, convenient online submission

- thorough peer review by experienced researchers in your field

- rapid publication on acceptance

- support for research data, including large and complex data types

- gold Open Access which fosters wider collaboration and increased citations

- maximum visibility for your research: over $100 \mathrm{M}$ website views per year

At BMC, research is always in progress.

Learn more biomedcentral.com/submissions 